\title{
Iron deficiency anemia with pica syndrome in 5 years old child
}

\author{
Nafea Hamoud Alanazi, Eyad Ahmed A Bohassan, Ahmed Fakhri Alsabi, Ridha Hussein A \\ Bohassan, Ali Hamoud N Alenazi \\ Pediatric Department, College of Medicine, Al-Jouf University \\ Corresponding author: Nafea Hamoud Alanazi, +966 56422 7398, E-mail: Dr.nafea1@ gmail.com
}

\begin{abstract}
:
Pica is an irregular want for and ingestion of either palatable or unpalatable substances. The condition has been distributed in medicinal diaries for quite a long time. The present case report is about a 5 years old female child reported to Department of General Pediatric with a chief complaint of high fever and constipation. The fever was progressive course for 2 days and constipation for 5 days. Furthermore, there was a history of abnormal behavior of ingestion of inedible substances like stones and sand. We seek through our report to shed the lights on the importance of reminding the physicians of diagnostic utility of pica side effects related with iron deficiency. As here in our case, if we ignored these diagnoses that caused pica, the child may suffer from serious complications such as very low hemoglobin, GIT abstraction, severe anemia and serious infection. Consequently, we concluded, that detailed medical and individual history of kids, helps in a superior determination and a proper treatment design.
\end{abstract}

Keywords: Pica, anemia, syndrome

\section{Introduction :}

Pica is an irregular want for and ingestion of either palatable or unpalatable substances. The condition has been distributed in medicinal diaries for quite a long time ${ }^{(1,2)}$. One of the primary cases of pica was noted in sixth century $\mathrm{AD}$ and was seen in a pregnant lady ${ }^{(1)}$. From that point forward, numerous instances of pica have been accounted for where patients have recognized ingesting ice 3D squares (pagophagia), clay (geophagia), dried pasta (amylophagia), chalk, starch, glue, kayexalate tar (resinphagia), tomatoes, lemons, cigarette butts, hair, lead, and clothing starch (for instance, Argo out of the container) ${ }^{(1,3)}$.

In spite of the fact that pica is most conspicuous in people with formative handicaps, it has been seen in people all things considered and ethnicity yet is more pervasive among the lower financial classes ${ }^{(2,4)}$. Around the world, $25 \%$ to $33 \%$ of all pica cases include little kids, $20 \%$ are pregnant ladies, and $10 \%$ to $15 \%$ are people with learning incapacities ${ }^{(5)}$. A little more percentage of patients have iron deficiency anemia. Pica postures huge health dangers that frequently require medical interventions. These patients are helpless to electrolyte and metabolic issues, lead and mercury poisoning, hypokalemia (from resinphagia), parasitic contaminations, tooth wear, intestinal impediment, and different issues of the gastrointestinal tract ${ }^{(6,7)}$. The correct etiology of pica stays vague, yet it is essentially connected with iron deficiency anemia ${ }^{(5,8)}$. As indicated by the 2001 World Health Organization (WHO) data, $30 \%$ of the kids aged between 0 and 4 years and $48 \%$ of the kids aged somewhere in the range of 5 and 14 years are anemic in developing nations ${ }^{(9)}$.

\section{Case presentation:}

The present case report is about a 5 years old female child reported to Department of General Pediatric with a chief complaint of high fever and constipation. The fever was progressive in course for 2 days and constipation for 5 days. Furthermore, there was a history of abnormal behavior of ingestion of inedible substances like stones and sand.

\section{On examination:}

The child looked unwell, febrile, very pale, no jaundice

Abdomen: tender, distended no organomegaly.

Eyes manifestation: pale conjunctiva

\section{Investigation:}

Laboratory test:

Complete blood count: HB: 4.9 g/dl, hematocrit: $21.1 \%, \mathrm{MCV}$ : 53.1 FL, WBC:9.88 $\times 10^{\wedge} 3 / U L$

Blood film: RBC: Anisocytosis with severe hypochromic, microcytosis and target cells.

Comment: Hypochromic microcytic anemia with lymphocytosis.

Serum iron: $3.08 \mathrm{mcg} / \mathrm{dl}$ and serum ferritin: $5.76 \mathrm{ng} / \mathrm{ml}$ 
Radiology findings:

\section{Figure 1. Abdominal X-ray:}

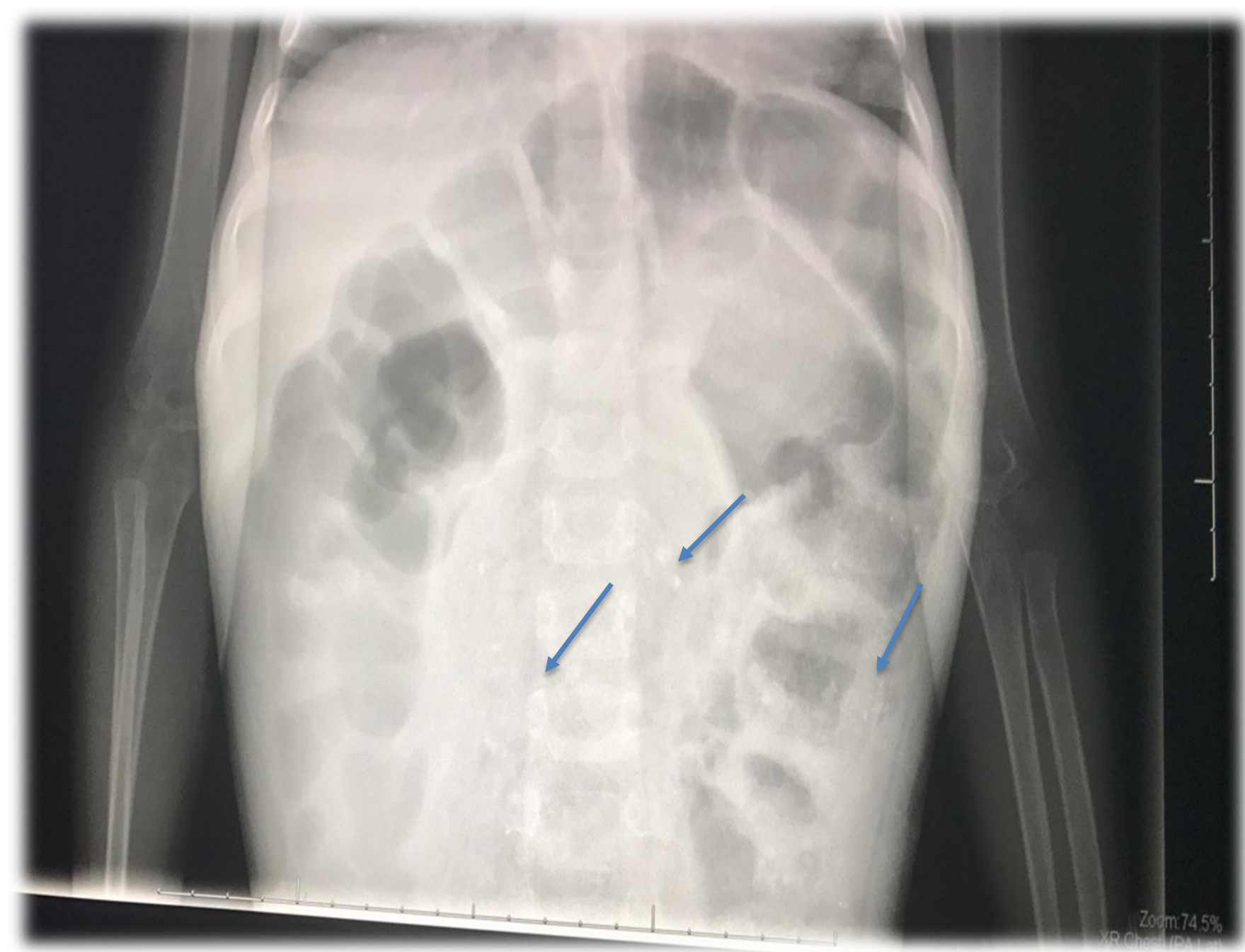

\section{Abdominal U/S:}

Dilated bowel loops, with significant dilatation of the rectum, containing multiple and abundant hyperechoic foci, mostly stones. Urinary bladder appears relatively high in position, mostly by the dilated rectum.

\section{Managements:}

IVF D5 $1 \frac{1}{2}$ Normal saline

Two unites packed RBCs

\section{Lactulose $2 \mathrm{ml} / \mathrm{Kg} / \mathrm{BW} /$ day orally}

Fleet enema

Discussion:

Albeit noticed since antiquity, pica remains a baffling and interesting occurrence. It is by all accounts firmly connected with iron deficiency anemia, and in the greater part of cases the uncommon eating and biting conduct vanishes upon iron supplementation ${ }^{(1,6,10,11)}$.
A few speculations exist concerning why iron deficiency causes pica, including physiological mechanisms; nonetheless, there is no single settled upon clarification ${ }^{(1,4)}$. Furthermore, pica has been connected to variables of gender, age, religion, culture, being under stress, nutritious deficiency and mental advancement (4)

At the point when related with iron deficiency, it is known to be a symptom of the deficiency rather than its reason ${ }^{8,12)}$. Incidentally, pica practices cause other nutritious deficiencies, for example, hypokalemia (clay, also, Kayexalate ingestion ${ }^{(5,13)}$. To explain, iron deficiency anemia (IDA) is known to be the furthermost basic nutritional deficiency worldwide and a noteworthy general medical issue especially in developing countries ${ }^{(14)}$. In any case, there is no unmistakable information 
about what number of people are affected by iron deficiency around the world, however, it is evaluated that ID is occurring in a large portion of the pre-school children and pregnant ladies and in developing countries no less than $30-40 \%$ when anemia is utilized as an incoordinate indicator of ID ${ }^{(8)}$.

We have viewed this case because it represents refined variations in the classical symptom multiplexes of pica. Apparently, the kids at first, had basic iron deficiency related with pica, including geophagia. The soil contained mixtures that bound both iron and zinc. Moreover, the secondary zinc deficiency caused the hepatomegaly and other strange abnormalities ${ }^{(15)}$. It is important to mention that pica is practiced when a patient is minimally managed. Patients are additionally obscure of their pica habits and are also frequently hesitant to specify it. Pica symptoms will accordingly go unnoticed except if the doctor particularly addresses them ${ }^{(12,16,17)}$. Prior finding of pica can demonstrate gainful particularly within the sight of an occult, bleeding malignancy or if the patient is pregnant ${ }^{(12)}$. However, pica in pregnancy isn't remarkable and, if unnoticed, may put both the mother and her fetus in danger ${ }^{(2,18)}$.

\section{Conclusion:}

We seek through our report to shed the lights on the importance of reminding the physicians of diagnostic utility of pica side effects related with iron deficiency. As here in our case, if we ignored these diagnoses that caused pica, the child may suffer from serious complications such as very low hemoglobin, GIT abstraction, severe anemia and serious infection. Consequently, we concluded, that detailed medical and individual history of kids, helps in a superior determination and a proper treatment design.

\section{References:}

1- Coltman CA (1969): Pagophagia and iron lack. JAMA., 207(3):513-516. https://jamanetwork.com/journals/jama/art icle-abstract $/ 343247$

2- Grotegut CA, Dandolu V，Katari S, Whiteman VE, Holtzman OG, and Teitelman M (2006): Baking soda pica: a case of hypokalemic metabolic alkalosis and rhabdomyolysis in pregnancy. Obstet Gynecol., 107:484-486.
3- Tisman G (1970): Resinphagia. N Engl J Med., 283(11):602.

4- Grivetti LE (1978): Culture, diet and nutrition: selected themes and topics. BioScience, 28(3):171-177.

5- Barker D (2005): Tooth wear as a result of pica. Br Dent J., 199:271-273.

6- Kathula SK (2008): Craving lemons: another form of pica in iron deficiency. Am J https://www.researchgate.net/publication/4 1941572_Pica_in_iron_deficiency_A_case series

7- Karnath BM (2004): Anemia in the adult patient. Hospital Physician, http://www.anesthesiaweb.org/images/ane mia/Karnath-2002.pdf

8- Kettaneh A, Eclache V, Fain O, Sontag C, Uzan M, Carbillon L, Stirnemann J, and Thomas $M$ (2005): Pica and food craving in patients with iron deficiency anemia: a case-control study in France. Am J Med., 118(2):185-188.

9- World Health Organization (2001): A guide for programme managers.Geneva, World Health Organization, 2001. Iron deficiency anaemia assessment, prevention, and control.

10- Kettaneh A, Eclache V, Fain O, Sontag C, Uzan M, Carbillon L, Stirnemann J, and Thomas $M$ (2005): Pica and food craving in patients with iron deficiency anemia: a casecontrol study in France. Am J Med., 118(2):185-188.

11- Pagophagia and Anemia (1969): Nutrition Reviews, 27(2):52-54.

12- Kushner RF, Gleason B and ShantaRetelny V (2004): Reemergence of pica following gastric bypass surgery for obesity: a new presentation of an old problem. J Am Diet Assoc., 104:1393-1397.

13- Ukaonu C, Hill A, and Christensen $F$ (2003): Hypokalemic myopathy in pregnancy caused by clay ingestion. Obstet Gynecol., 102(5):1169-1171.

14- Yasir K, and Glenn $T$ (2010): Pica in iron deficiency: a case series. Khan and Tisman Journal of Medical Case Reports, 4:86 
15- Say B, Ozsoylu S and Berkel I (1969): Geophagia associated with iron-deficiency anemia, hepatosplenomegaly, hypogonadism and dwarfism. A syndrome probably associated with zinc deficiency. Clin Pediatr (Phila), 8(11):661-8.

16- Falcomata $T$, Roane $H$, and Pabico $R$ (2007): Unintentional stimulus control during the treatment of pica displayed by a young man with autism. RASD., 350:3
17- Scully R, Mark E, McNeely W, Ebeling $S$ (1998): Case records of the

Massachusetts General Hospital: case 37. N Engl J Med., 339(24):1766-1772.

18- Lopez L, Langini S, Pita de Portela M (2007): Maternal iron status and neonatal outcomes in women with pica during pregnancy. Int J Gynecol

Obstet., 98:151-152. 